\title{
Disc Rehydration after Dynamic Stabilization: A Report of 59 Cases
}

\author{
Atilla Yilmaz ${ }^{1}$, Salim Senturk ${ }^{2}$, Mehdi Sasani ${ }^{2}$, Tunc Oktenoglu' ${ }^{2}$, \\ Onur Yaman ${ }^{2}$, Hakan Yildirim³ ${ }^{3}$ Tuncer Suzer ${ }^{2}$, Ali Fahir Ozer ${ }^{2}$ \\ ${ }^{1}$ Department of Neurosurgery, Mustafa Kemal University, Hatay, Turkey \\ ${ }^{2}$ Department of Neurosurgery, Koc University School of Medicine, Istanbul, Turkey \\ ${ }^{3}$ Department of Radiology, American Hospital, Istanbul, Turkey
}

Study Design: A retrospective study investigating decrease in the nucleus pulposus signal intensity or disc height on magnetic resonance imaging (MRI) and disc degeneration.

Purpose: Although a degenerated disc cannot self-regenerate, distraction or stabilization may provide suitable conditions for rehydration and possible regeneration. This study aimed to evaluate clinical outcomes and disc regeneration via MRI in a series of patients with degenerative disc disease (DDD) who underwent lumbar stabilization with a dynamic stabilization system (DSS).

Overview of Literature: A dynamic system provides rehydration during early DDD.

Methods: Fifty-nine patients (mean age, 46.5 years) who undedwent stabilization with DSS for segmental instability (painful black disc) between 2004 and 2014 were retrospectively evaluated. All patients underwent MRI preoperatively and 12 months postoperatively. Intervertebral disc (IVD) degeneration grades at the implanted segment were categorized using the Pfirrmann classification system. Patients were followed for a mean of 6.4 years, and clinical outcomes were based on visual analog scale (VAS) and Oswestry disability index (ODI) scores.

Results: Significant improvements in back pain VAS and ODI scores from before surgery (7 and 68\%, respectively) were reported at 6 ( 2.85 and $27.4 \%$, respectively) and 12 months postoperatively $(1.8$ and $16.3 \%$, respectively). Postoperative IVD changes were observed in 28 patients. Improvement was observed in 20 patients (34\%), whereas progressive degeneration was observed in eight patients (13.5\%). Thirty-one patients (52.5\%) exhibited neither improvement nor progression. Single Pfirrmann grade improvements were observed in $29 \%$ of the patients and two-grade improvements were observed in $5 \%$.

Conclusions: Our observations support the theory that physiological movement and a balanced load distribution are necessary for disc regeneration. We conclude that DSS may decelerate the degeneration process and appears to facilitate regeneration.

Keywords: Intervertebral disc degeneration; Instrumentation; Regeneration; Rehydration

\section{Introduction}

The intervertebral disc (IVD) is the most important sta- bilizing and load-bearing structure in the spinal column and is also among the main causes of back pain [1]. IVD consists of two parts: the annulus fibrosus (AF) and the

\footnotetext{
Received Jun 2, 2016; Revised Oct 5, 2016; Accepted Oct 28, 2016

Corresponding author: Ali Fahir Ozer

Department of Neurosurgery, Koc University School of Medicine, Rumelifeneri Yolu Sariyer, Istanbul 34450, Turkey

Tel: +90-212-338-1401, Fax: +90-212-338-1559, E-mail: alifahirozer@gmail.com
} 
nucleus pulposus (NP). AF comprises fibrocartilaginous and fibrous connective tissues, whereas NP primarily consists of proteoglycans. In IVDs, most fibrils comprise types 1 and 2 collagens, and aggrecan is the dominant proteoglycan [2]. Proteoglycans, which are hydrophilic in nature, maintain adequate disc turgor [3], thereby allowing the radial distribution of compressive loads [4].

Disc degeneration is associated with a loss of proteoglycans [5], leading to a decrease in the osmotic pressure of the disc matrix and a subsequent loss of hydration. Because a dehydrated IVD cannot uniformly distribute a compressive load, the load is, therefore, applied to posterior elements. Disc degeneration also causes losses in disc height and affects other spinal structures. Compression of spinal neural structures, the degenerative facets, or the disc itself can cause pain [6]. Magnetic resonance imaging (MRI) assesses the fluid contents of soft tissues. In the spine, the fluid content of NP is reflected by its MRI signal intensity. Decreases in this intensity or disc height, which are associated with disc degeneration, can be clearly identified by a T2-weighted MRI evaluation [7].

A degenerated disc cannot self-regenerate. In addition, long-term elevated or impaired intradiscal pressure prevents regeneration $[8,9]$. However, distraction or stabilization may provide suitable conditions for rehydration and, therefore, regeneration $[10,11]$. Several studies have suggested that dynamic stabilization systems (DSSs) can provide these suitable conditions $[10,12,13]$. Therefore, our study aimed to evaluate clinical outcomes and disc regeneration via MRI in a series of patients with degenerative disc disease (DDD) who underwent lumbar stabilization with DSS.

\section{Materials and Methods}

\section{Study design}

Fifty-nine patients (33 females and 26 males) with segmental instability and DDD (painful black disc) who underwent a DSS procedure between 2004 and 2014 and participated in follow-up MRI scans were included in the study. All patients were stabilized after annulus reconstruction, which involved the removal of dead nuclear tissue fragments from among the annulus layers, followed by bipolar cauterization and shrinkage of the remaining slack annulus layers and strengthening of the posterior wall. Patients had a mean age of 46.5 years (range, 27-67 years)
Table 1. Demographic data of the 59 patients

\begin{tabular}{|c|c|}
\hline Value & Number \\
\hline \multicolumn{2}{|l|}{ Sex } \\
\hline Female & 33 \\
\hline Male & 26 \\
\hline Age (yr) & 46.5 (range, 27-67) \\
\hline \multicolumn{2}{|l|}{ Complaint } \\
\hline Back pain & $59 / 59$ \\
\hline Mild leg pain & $22 / 59$ \\
\hline \multicolumn{2}{|l|}{ Neurologic examination } \\
\hline $\begin{array}{l}\text { Paravertebral muscle spasm, } \\
\text { bilateral positive }\end{array}$ & $59 / 59$ \\
\hline Limited flexion/extension at low back & $59 / 59$ \\
\hline (+) Laseque test & $22 / 59$ \\
\hline \multicolumn{2}{|l|}{ Operation } \\
\hline $\mathrm{L} 2-\mathrm{L} 3$ & $1 / 59$ \\
\hline L3-L4 & $4 / 59$ \\
\hline $\mathrm{L} 4-\mathrm{L} 5$ & $40 / 59$ \\
\hline L5-S1 & $14 / 59$ \\
\hline
\end{tabular}

and were followed up for a mean of 6.4 years (range, 1-11 years). Patients' demographic data are shown in Table 1.

The main indications for surgical decisions included a black disc on MRI, axial low back pain (DDD) with or without disc herniation, modic types I and II degeneration, and large annulus defects. The last group included patients with painful discs and severe fatty degeneration or paravertebral muscle atrophy. Although no useful measurement of muscle strength is currently available, MRI can provide valuable information about fatty degeneration and volumes of back muscles [14]. The study exclusion criteria included absolute spinal stenosis, spondylolisthesis, vertebral fractures, severe osteoporosis, and the presence of malignancy or infection. Patients with a history of previous spinal surgery were also excluded.

\section{Radiological examinations}

MRI was performed on a 1-T scanner (Siemens Magnetom Espree; Siemens AG, Erlangen, Germany), using the following parameters for sagittal plain T2-weighted imaging: repetition time, 3,820 millieconds; echo time, 112 milliseconds; and slice thickness, $4 \mathrm{~mm}$. For all studies, the imaging protocol comprised T1- and T2weighted sagittal and T2-weighted axial fast spin-echo 
sequences. All patients underwent MRI preoperatively and 12 months postoperatively. MRI scans were evaluated by three independent radiologists, and IVD degeneration grades were assigned at the implanted segment according to the Pfirrmann classification of lumbar IVD degeneration at the implanted segment [15].

\section{Clinical evaluation}

The patients' quality of life and back pain scores were evaluated using the Oswestry disability index (ODI) and visual analog pain scale (VAS; 0 , no pain; 10 , worst pain), respectively, preoperatively and at 6 and 12 months postoperatively.

\section{Surgical technique}

For patients with a loose annulus without rupture, we only performed a dynamic stabilization procedure via the Wiltse approach. For patients in whom disc fragments were present among the ruptured annulus layers, we removed the fragments under microscopic guidance before performing stabilization. All operations were performed under general anesthesia and with the patient in a kneeto-chest position to maintain physiological lordosis of the lumbar vertebrae. A prophylactic antibiotic was preoperatively administered to all patients. The pathological level was localized using intraoperative fluoroscopy. An approximately $3-\mathrm{cm}$ medial incision was made if annulus reconstruction was required. After paravertebral musculature dissection, a small laminectomy was performed using a high-speed drill. Subsequently, the ligamentum flavum was incised near the degenerated disc. After identifying the dura and nerve root and determining the annulus, a small incision was immediately made over the ruptured annulus, and small fragments were removed using a micro-nerve hook. The annulus was then cauterized and made to shrink with a bipolar cautery.

Following microscopic decompression, posterior dynamic transpedicular stabilization was performed via the Wiltse approach [16]. Manual dissection was used to open a path between the multifidus and longissimus muscles and reveal the transverse processes, and pedicle screws were then inserted under lateral fluoroscopic (C-arm) visualization. Facet joint preservation was ensured. The spinal lordosis angle was assessed before rod implantation, and all efforts were made to maintain normal physiologi- cal alignment. After meticulous hemostasis, the wound was closed in a layered fashion.

Two different stabilization systems were used. The Cosmic DSS (Ulrich GmbH \& Co. KG, Ulm, Germany) was used in 30 patients, and the Safinaz DSS (Medikon AS, Istanbul, Turkey) was used in 29 patients. The Cosmic system used a $6.25-\mathrm{mm}$ threaded rigid rod, with a hinged screw head to limit movement. The Safinaz system used a 6-mm non-threaded rigid rod, with a hinged screw head to limit movement. Stabilization was performed at the following levels: L2-L3 in one patient, L3-L4 in four, L4L5 in 40, and L5-S1 in 14. All surgeries were performed by the same two experienced surgical teams, and optimal neural decompression and physiological motion preservation were the goals for all patients.

\section{Results}

No adhesion, fibrosis, infection, or chronic inflammation was observed during the long-term follow-up period. Similarly, no disc herniation was observed.

\section{VAS and ODI scores}

The back pain VAS scores significantly improved from 7 (range, 6-9) before DSS procedures to 2.85 (range, 1-5) at 6 months and 1.8 (range, $0-3$ ) at 12 months postoperatively. Similarly, the ODI scores significantly improved from $68 \%$ (range, $48 \%-92 \%$ ) preoperatively to $27.4 \%$ (range, $12 \%-56 \%$ ) at 6 months and $16.3 \%$ (range, $4 \%-$ $32 \%)$ at 12 months postoperatively (Table 2 ).

\section{MRI}

We compared disc degeneration at the implantation segments on preoperative and 1-year postoperative T2weighted sagittal MRI scans according to the Pfirrmann lumbar IVD degeneration classification. In the preoperative images, we observed grades 3,4 , and 5 degeneration in 7, 45, and 7 patients, respectively. After 12 months of follow-up, we observed grades $2,3,4$, and 5 degeneration in $7,19,19$, and 14 patients, respectively (Table 3 ).

Postoperative IVD changes were observed in 28 patients. Improvement (disc rehydration) was observed in 20 patients (34\%) (Fig. 1), including single-grade improvement in 17 patients (29\%) and two-grade improvement in three patients (5\%), whereas progressive degeneration 
Table 2. Clinical outcome

\begin{tabular}{|c|c|c|c|c|c|c|}
\hline \multirow{2}{*}{ Outcome } & \multicolumn{2}{|c|}{ Preoperative } & \multicolumn{2}{|c|}{ Postoperative 6 mo } & \multicolumn{2}{|c|}{ Postoperative 12 mo } \\
\hline & VAS & ODI & VAS & ODI & VAS & ODI \\
\hline Rehydration (n=20) & $7.50 \pm 0.82$ & $68.10 \pm 10.94$ & $2.80 \pm 1.15$ & $30.2 \pm 8.98$ & $1.65 \pm 0.74$ & $13.30 \pm 5.84$ \\
\hline Progressive degeneration $(n=8)$ & $6.75 \pm 0.89$ & $68.25 \pm 9.65$ & $2.87 \pm 0.64$ & $26.00 \pm 7.41$ & $2.25 \pm 0.46$ & $17.25 \pm 7.78$ \\
\hline No changes $(n=31)$ & $7.12 \pm 0.84$ & $68.80 \pm 9.44$ & $2.80 \pm 0.87$ & $28.10 \pm 9.84$ & $1.58 \pm 0.99$ & $16.06 \pm 7.25$ \\
\hline Mean $(n=59)$ & $7.20 \pm 0.86$ & $68.50 \pm 9.83$ & $2.81 \pm 0.93$ & $28.57 \pm 9.22$ & $1.69 \pm 0.87$ & $15.28 \pm 6.92$ \\
\hline
\end{tabular}

VAS, visual analog scale; ODI, Oswestry disability index.

Table 3. Comparison of disc signal change between preoperative and postoperative period

\begin{tabular}{lccccc} 
Patient evaluation & Grade 1 & Grade 2 & Grade 3 & Grade 4 & Grade 5 \\
Preoperative & 0 & 0 & 7 & 45 & 7 \\
Postoperative & 0 & 7 & 19 & 19 & 14 \\
\hline
\end{tabular}

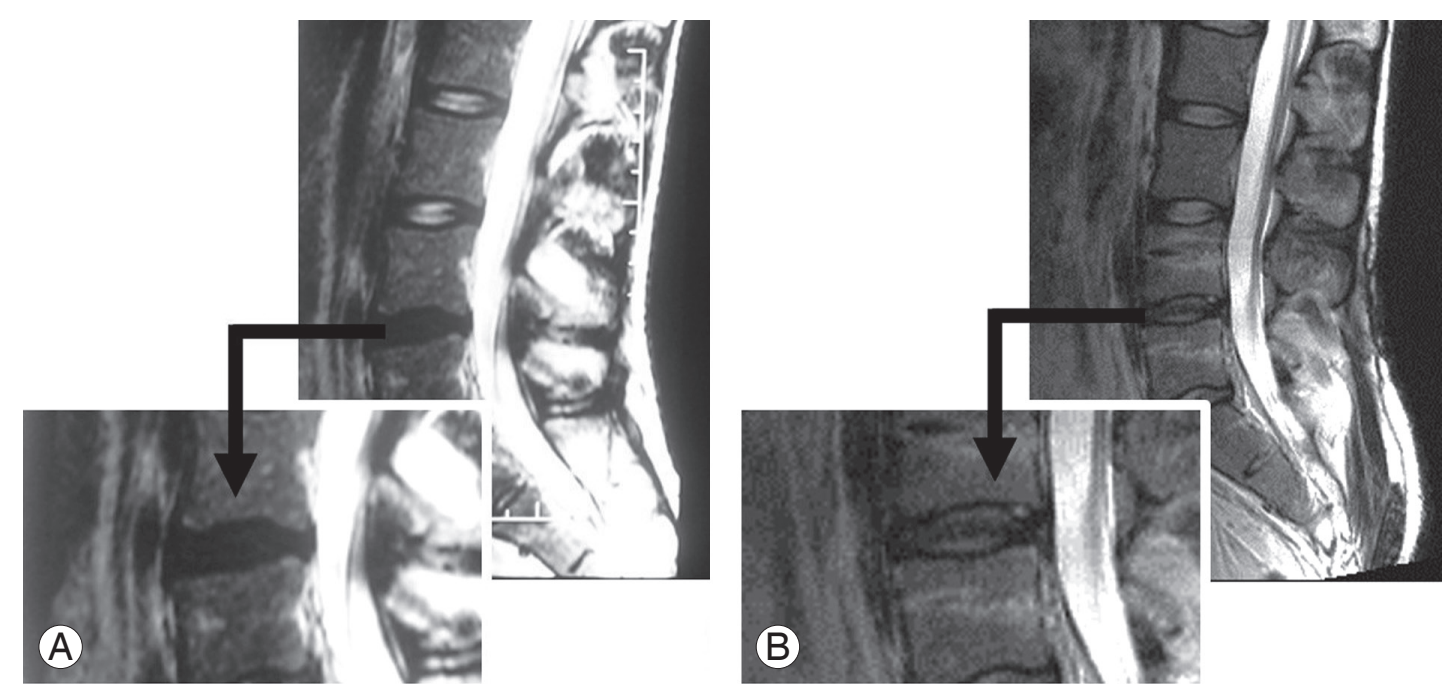

Fig. 1. Representative case of rehydration. A patient with severe back pain with attacks three or four times a year. The patient has painfull degenerative disc disease. (A) Preoperative T2-weighted (T2W) magnetic resonance imaging (MRI). Decreased signal intensity in the disc space at the $L 4-L 5$ level (Pfirrmann grade 4). (B) Postoperative T2W MRI. An increase in signal intensity indicative of rehydration is observed at the same level after 12 months (Pfirrmann grade 2).

was observed in eight patients (13.5\%) (Fig. 2). Thirtyone patients (52.5\%) exhibited neither improvement nor progression (Figs. 3, 4).

Progressive degeneration was observed only in patients with grade 4 degeneration, and recurrent disc herniation was not observed in any patients. There were no clinical findings associated with adjacent segment disease. On the other hand, several patients exhibited adjacent segment alterations after undergoing stabilization procedures. Among patients without disc changes $(n=31)$, only one developed degeneration at an adjacent segment. Among those with progressive degeneration $(n=8)$, two patients developed adjacent segment degeneration. None of the patients with improved conditions $(n=20)$ developed alterations at adjacent segments.

\section{Discussion}

A decreases in the fluid content of NP leads to a decrease in hydrostatic pressure [7], which can directly affect the 


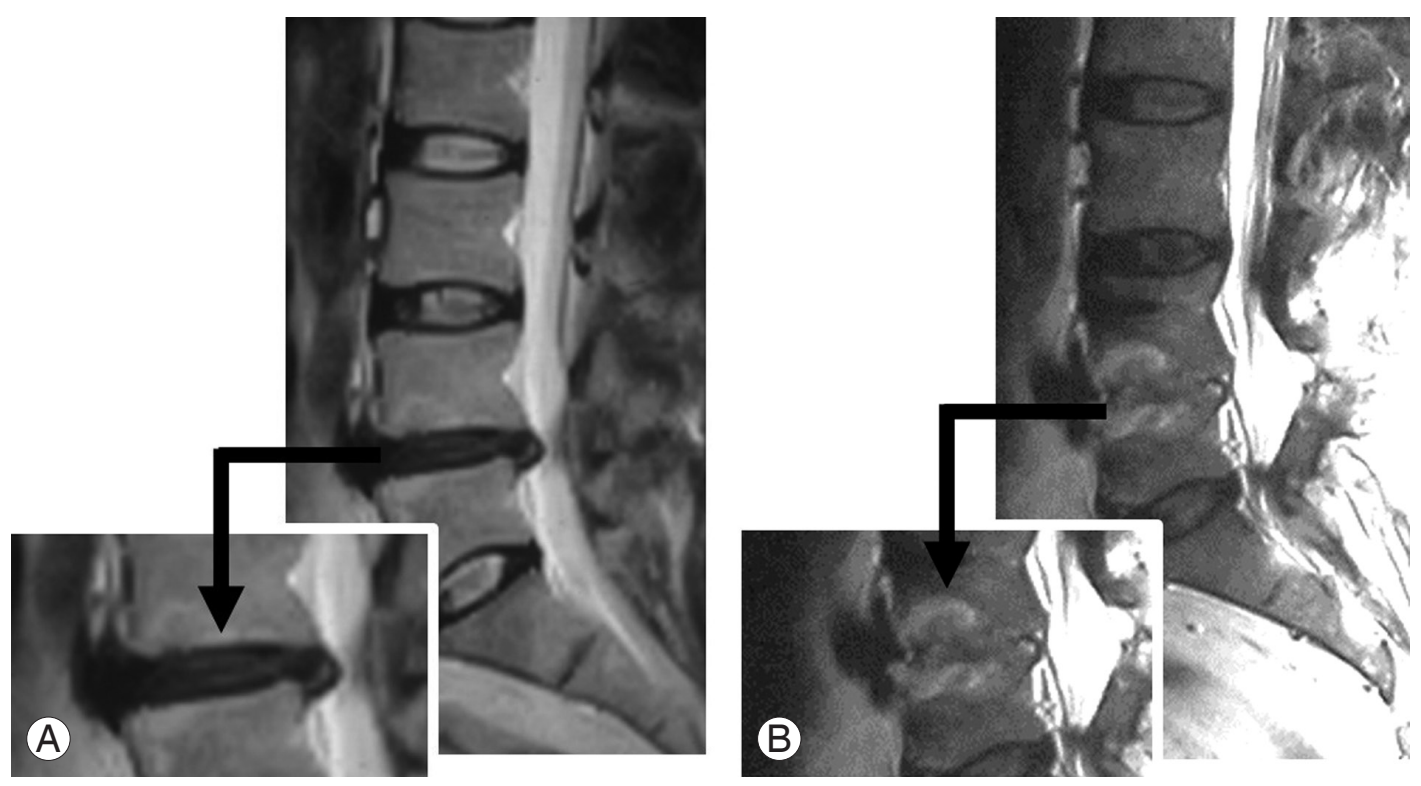

Fig. 2. Representative case of fusion in a female patient with back pain caused by an extensive posterior annulus rupture. (A) Preoperative T2-weighted (T2W) magnetic resonance imaging (MRI). Decreased signal intensity in the disc space at the L4L5 level (Pfirrmann grade 3). (B) Postoperative T2W MRI. Fusion at the same level is visible after 12 months postoperatively (Pfirrmann grade 5).
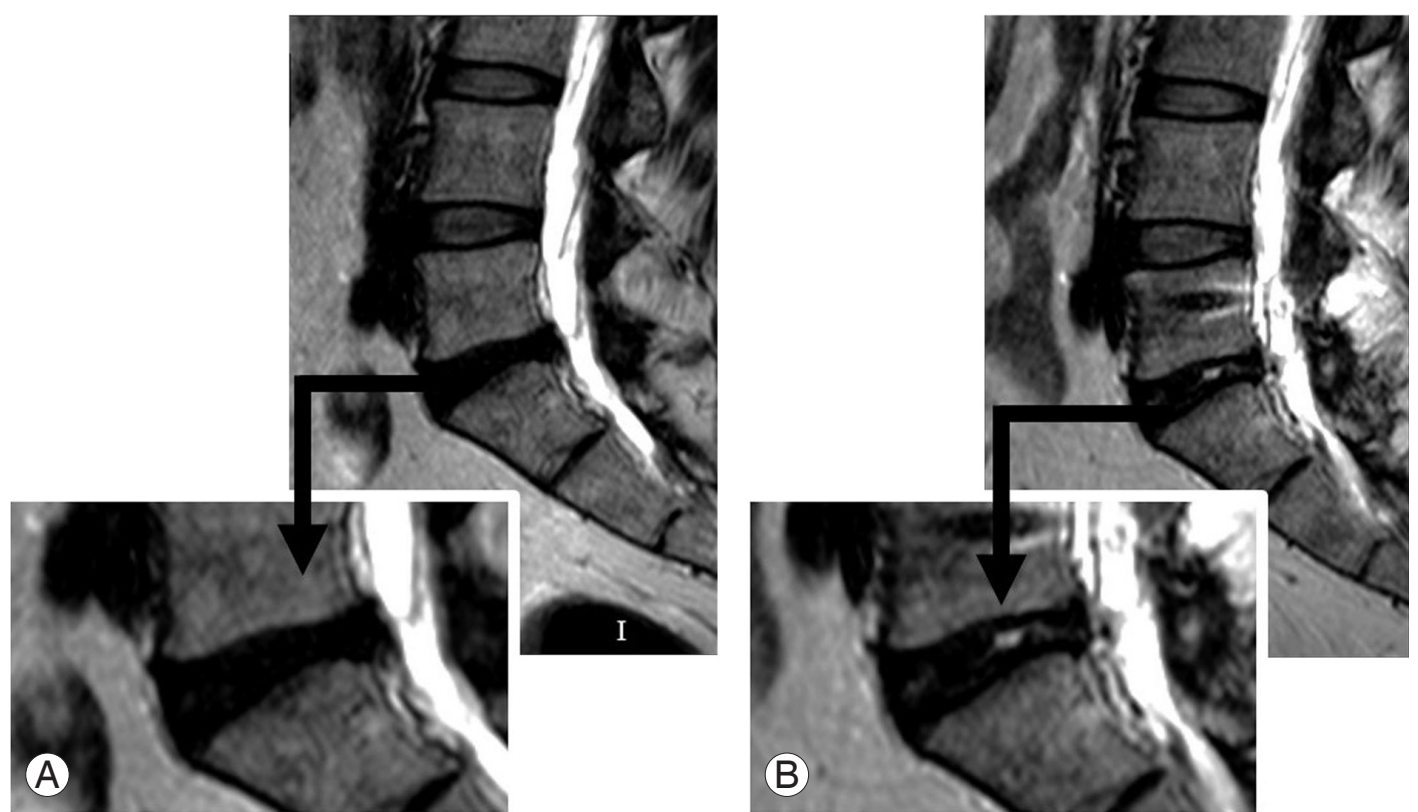

Fig. 3. Representative case of no change in a male patient with severe, chronic episodes of back pain and recent leg pain caused by a foraminal disc herniation. (A) Preoperative T2-weighted (T2W) magnetic resonance imaging (MRI). Decreased signal intensity in the disc space at the L5-S1 level (Pfirrmann grade 4). (B) Postoperative T2W MRI. No change in signal intensity at the same level after 12 months postoperatively (Pfirmann grade 4).

degeneration and regeneration cascade [8]. The inability of a degenerative disc to uniformly distribute a load can lead to pain and other effects on anatomical spinal structures [17] and may cause facet arthrosis, spinal stenosis, instability, and severe disability over time [18]. Furthermore, a decreased proteoglycan content may lead to increased neural growth and neovascularization in the disc, which can also cause pain [19]. Moreover, instability 


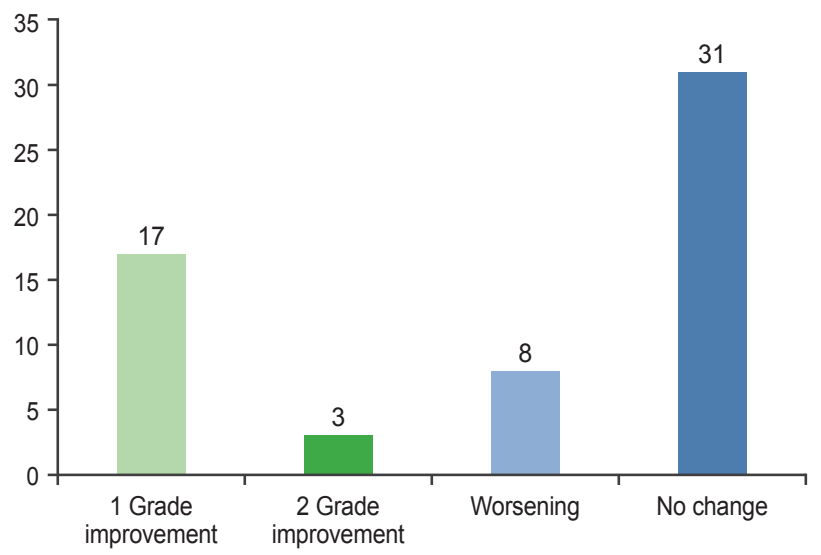

Fig. 4. Disc signal change between preoperative and postoperative period.

and non-physiological movement can also accelerate the degeneration cascade [20].

Multiple studies have shown that axial IVD distraction can induce extracellular matrix gene expression [11] and affect disc nutrition via fluid movement [21]. However, axial distraction can only be achieved via stabilization systems. Patients with simultaneous segmental instability and disc herniation are well known to be at a risk of recurrent disc herniation. Although these patients may be treated via microdiscectomy alone, we performed lumbar dynamic stabilization in addition to microdiscectomy for such patients.

Lumbar fusion surgery is widely used to treat degenerative lumbar diseases, such as instability or stenosis [22]. In a cadaveric study, Schmoelz et al. [23] found that measurements of intradiscal pressure at bridged segments did not reveal differences between dynamic and other systems. However, physiological movement and a balanced load distribution must be maintained to allow disc regeneration. It is known that although a degenerated disc cannot self-regenerate, various stabilization systems may provide the circumstances required for regeneration [24]. Several studies have suggested that compared with decompression alone, the combination of this procedure with fusion led to improved patient outcomes [25]. However, rigid internal fixation and fusion can also increase the incidence of adjacent segment disease. Dynamic systems can prevent adjacent segment pathology by preserving physiological movement [26]. Schnake et al. [13] suggested that DSS could maintain physiological motion and optimal intradiscal pressure and, therefore, prevent IVD degeneration while providing optimal conditions for disc regeneration. Currently, in our study, we cannot claim that rehydration had a positive effect on the patients' clinical conditions as these patients had also undergone surgical instrumentation. We could only observe the discs after instrumentation and comment on the relationship between the degeneration degree and final radiological results. However, we could also evaluate the adjacent segments in our patients. In this study, we observed no apparent changes in the rehydration group, which is an encouraging finding. Radiologically, adjacent segment disease was mostly observed in the progressive degeneration group, which is an acceptable result.

DSS is a relatively new form of stabilization for disc degeneration or instability [25]. DSSs can share the load between two spinal columns and provide load-sharing and load-bearing features while preserving physiological motion. Thus, these systems can compensate nonphysiological loads and limit pathological movement $[20,26]$, thereby providing a suitable environment for rehydration and even regeneration. Several clinical studies have suggested that DSSs can prevent the progression of degeneration at the bridged level after discectomy and decompression $[27,28]$. However, DSSs have not previously been widely used, and relevant published data are limited. Several studies have reported inconsistent results regarding clinical outcomes and radiographical changes. Moreover, a few patients have experienced fusion after dynamic stabilization, despite the use of hinged screws for movement protection.

Mulholland and Sengupta [29] claimed that the main cause of low back pain is an unbalanced load distribution across the disc after disc degeneration, rather than an increased range of motion. However, abnormal translational or directional motion unbalances the load distribution over the disc and, thus, causes pain. They also stated that the main aim of a stabilization device is to protect motion and restrict abnormal motion [29]. Several DSSs are currently available, including the Safinaz (Medikon AS), Dynesys (Zimmer), Cosmic (Ulrich GmbH \& Co. KG), Graf (Neoligaments), and FASS (AO International) systems. Only the Safinaz and Cosmic systems were used in the present study.

Lotz and Chin [9] observed extensive cell death in NP and AF after long periods of high stress, as well as an increase in the number of apoptotic cells with increases in stress and time. Kasra et al. [8] showed that an extensive short-term load may stimulate protein synthesis, whereas 
prolonged loading led to protein degradation and inhibited protein synthesis in annulus cells. Sengupta and Mulholland [12] claimed that DSS could create a suitable environment for growth factor application and stem cell disc regeneration. These cell culture studies illustrate the importance of hydrostatic pressure and stabilization with regard to IVD degeneration.

Through animal studies, Guehring et al. [11] and Kroeber et al. [20] demonstrated improvements in disc health via external spinal instrumentation and also demonstrated that compressive loads could lead to IVD degeneration. However, subsequent stabilization can stop or even reverse this process $[11,20]$. In their in vivo study of New Zealand White rabbits, they used a custom-made device to apply a compression load to IVD and thus generate degeneration over a 28-day period. At the end of the compression period, the authors applied DSS with an external device to a subgroup of these rabbits for another 28 days. After distraction, the authors observed increases in disc height and MRI signal intensity, a decrease in apoptotic cell count, and improvement in annulus morphology.

Putzier et al. [27] compared patients who underwent discectomy alone with those who underwent discectomy with stabilization. After a 34-month follow-up period, no progressive degeneration was observed in the latter group. However, Kumar et al. [24] reported a 56\% radiographical degeneration rate among segments bridged with a dynamic system. Our results were not entirely consistent with the latter study as we observed progressive radiographical degeneration at the bridged level in only $12 \%$ of the patients.

Cho et al. [10] published a case report of obvious disc regeneration visualized on MRI at 1 year after a DSS procedure. Bordes-Monmeneu et al. [30] reported rehydration in nine of $20(45 \%)$ patients in a 9-month followup period after dynamic stabilization. Similarly, in our study, we observed rehydration in 34 of 55 patients (34\%), with single-grade improvement in $29 \%$ and two-grade improvement in 5\% of the patients (Pfirrmann classification). This observation supports the theory that physiological movement maintenance and a balanced load distribution are necessary for disc regeneration.

The application of DSS to a functional segment does not guarantee that rehydration of the disc will occur as the degeneration level is a very important factor in healing. During early degeneration, this process may be reversible, as observed in some of our patients. However, fusion is inevitable in patients with advanced degeneration, just as spontaneous fusion occurs with end-stage degeneration in nonoperative patients. A limited discectomy should be performed in patients with Pfirrmann grade 1-2 degeneration because of the high risk of recurrence. It is likely that aggressive subtotal discectomy led to fusion in the patient shown in Fig. 2.

Our study was mainly limited by the short follow-up period. A longer follow-up period may provide more consistent conclusions. In addition, further randomized clinical trials are needed to prove that DSSs can facilitate disc regeneration.

\section{Conclusions}

Our results suggest that DSSs do not harm IVD and appear to facilitate regeneration, with benefits to the patient. We conclude that there are three possible results after DSS application: (1) disc rehydration, (2) no change, and (3) fusion. We observed no relationship between radiological and clinical results.

\section{Conflict of Interest}

No potential conflict of interest relevant to this article was reported.

\section{References}

1. Hickey DS, Hukins DW. X-ray diffraction studies of the arrangement of collagenous fibres in human fetal intervertebral disc. J Anat 1980;131(Pt 1):81-90.

2. Zhao CQ, Wang LM, Jiang LS, Dai LY. The cell biology of intervertebral disc aging and degeneration. Ageing Res Rev 2007;6:247-61.

3. Hukins DW. A simple model for the function of proteoglycans and collagen in the response to compression of the intervertebral disc. Proc Biol Sci 1992;249:281-5.

4. Huang RC, Wright TM, Panjabi MM, Lipman JD. Biomechanics of nonfusion implants. Orthop Clin North Am 2005;36:271-80.

5. Lyons G, Eisenstein SM, Sweet MB. Biochemical changes in intervertebral disc degeneration. Biochim Biophys Acta 1981;673:443-53.

6. Coppes MH, Marani E, Thomeer RT, Groen GJ. Innervation of "painful" lumbar discs. Spine (Phila Pa 
1976) $1997 ; 22: 2342-9$.

7. Haughton V. Medical imaging of intervertebral disc degeneration: current status of imaging. Spine (Phila Pa 1976) 2004;29:2751-6.

8. Kasra M, Goel V, Martin J, Wang ST, Choi W, Buckwalter J. Effect of dynamic hydrostatic pressure on rabbit intervertebral disc cells. J Orthop Res 2003;21:597-603.

9. Lotz JC, Chin JR. Intervertebral disc cell death is dependent on the magnitude and duration of spinal loading. Spine (Phila Pa 1976) 2000;25:1477-83.

10. Cho BY, Murovic J, Park KW, Park J. Lumbar disc rehydration postimplantation of a posterior dynamic stabilization system. J Neurosurg Spine 2010;13:57680.

11. Guehring T, Omlor GW, Lorenz H, et al. Disc distraction shows evidence of regenerative potential in degenerated intervertebral discs as evaluated by protein expression, magnetic resonance imaging, and messenger ribonucleic acid expression analysis. Spine (Phila Pa 1976) 2006;31:1658-65.

12. Sengupta DK, Mulholland RC. Fulcrum assisted soft stabilization system: a new concept in the surgical treatment of degenerative low back pain. Spine (Phila Pa 1976) 2005;30:1019-29.

13. Schnake KJ, Putzier M, Haas NP, Kandziora F. Mechanical concepts for disc regeneration. Eur Spine J 2006;15 Suppl 3:S354-60.

14. Carragee EJ, Han MY, Suen PW, Kim D. Clinical outcomes after lumbar discectomy for sciatica: the effects of fragment type and anular competence. J Bone Joint Surg Am 2003;85:102-8.

15. Pfirrmann CW, Metzdorf A, Zanetti M, Hodler J, Boos N. Magnetic resonance classification of lumbar intervertebral disc degeneration. Spine (Phila $\mathrm{Pa}$ 1976) 2001;26:1873-8.

16. Wiltse LL, Spencer CW. New uses and refinements of the paraspinal approach to the lumbar spine. Spine (Phila Pa 1976) 1988;13:696-706.

17. Adams MA, McNally DS, Dolan P. 'Stress' distributions inside intervertebral discs: the effects of age and degeneration. J Bone Joint Surg Br 1996;78:965-72.

18. Urban JP, Roberts S. Degeneration of the intervertebral disc. Arthritis Res Ther 2003;5:120-30.

19. Freemont AJ, Peacock TE, Goupille P, Hoyland JA, O’Brien J, Jayson MI. Nerve ingrowth into diseased intervertebral disc in chronic back pain. Lancet 1997;350:178-81.

20. Kroeber M, Unglaub F, Guehring T, et al. Effects of controlled dynamic disc distraction on degenerated intervertebral discs: an in vivo study on the rabbit lumbar spine model. Spine (Phila Pa 1976) 2005;30:181-7.

21. Urban JP, Smith S, Fairbank JC. Nutrition of the intervertebral disc. Spine (Phila Pa 1976) 2004;29:27009.

22. Bono CM, Kadaba M, Vaccaro AR. Posterior pedicle fixation-based dynamic stabilization devices for the treatment of degenerative diseases of the lumbar spine. J Spinal Disord Tech 2009;22:376-83.

23. Schmoelz W, Huber JF, Nydegger T, Claes L, Wilke HJ. Influence of a dynamic stabilisation system on load bearing of a bridged disc: an in vitro study of intradiscal pressure. Eur Spine J 2006;15:1276-85.

24. Kumar A, Beastall J, Hughes J, et al. Disc changes in the bridged and adjacent segments after Dynesys dynamic stabilization system after two years. Spine (Phila Pa 1976) 2008;33:2909-14.

25. Kaner T, Ozer AF. Dynamic stabilization for challenging lumbar degenerative diseases of the spine: a review of the literature. Adv Orthop 2013;2013:753470.

26. Ozer AF, Crawford NR, Sasani M, et al. Dynamic lumbar pedicle screw-rod stabilization: two-year follow-up and comparison with fusion. Open Orthop J 2010;4:137-41.

27. Putzier M, Schneider SV, Funk JF, Tohtz SW, Perka C. The surgical treatment of the lumbar disc prolapse: nucleotomy with additional transpedicular dynamic stabilization versus nucleotomy alone. Spine (Phila Pa 1976) 2005;30:E109-14.

28. Schnake KJ, Schaeren S, Jeanneret B. Dynamic stabilization in addition to decompression for lumbar spinal stenosis with degenerative spondylolisthesis. Spine (Phila Pa 1976) 2006;31:442-9.

29. Mulholland RC, Sengupta DK. Rationale, principles and experimental evaluation of the concept of soft stabilization. Eur Spine J 2002;11 Suppl 2:S198-205.

30. Bordes-Monmeneu M, Bordes-Garcia V, RodrigoBaeza F, Saez D. System of dynamic neutralization in the lumbar spine: experience on 94 cases. Neurocirugia (Astur) 2005;16:499-506. 